\title{
INVESTIGACIONES
}

\section{La inmigración desde la mirada del profesor de matemáticas del sur de Chile}

\author{
Immigration from the perspective of the teacher of mathematics \\ in southern Chile
}

\author{
Elizabeth H. Arredondo ${ }^{a}$, Maximina Márquez Torres ${ }^{a}$, Jaime I. García-García ${ }^{a}$ \\ ${ }^{a}$ Universidad de Los Lagos, Osorno, Chile. \\ elizabeth.hernandez@ulagos.cl,maximina.marquez@ulagos.cl,jaime.garcia@ulagos.cl
}

\begin{abstract}
RESUMEN
El propósito de este artículo es exponer, desde la mirada de dos profesores de matemáticas del sur de Chile, el fenómeno de encuentro y reconocimiento que hoy vive la población inmigrante en el ámbito de las matemáticas escolares, bajo tres amplias categorías de códigos sociolingüísticos instalados en el discurso del profesor y la relación de estos con el currículo; a saber, el empoderamiento, la escolaridad inmigrante y el fracaso escolar. Este estudio de tipo exploratorio, descriptivo e interpretativo, con un toque microetnográfico, se apoya en la Teoría de los Códigos Sociolingüísticos de Bernstein para observar las tensiones existentes entre la política nacional que apertura la educación a esta población inmigrante y la falta de preparación de los profesores para afrontar los cambios culturales en el aula de matemáticas; develando que una de las barreras que tienen estos estudiantes es el establecimiento de una comunicación efectiva en el medio escolar.
\end{abstract}

Palabras claves: Escolaridad inmigrante, empoderamiento, fracaso escolar, matemáticas escolares.

\begin{abstract}
The purpose of this article is to expose, from the perspective of two mathematics teachers from southern Chile, the phenomenon of encounter and recognition lived today by the immigrant population in the field of school mathematics, under three broad categories of sociolinguistic codes installed in the professor's discourse and their relationship with the curriculum, namely, the empowerment, immigrant schooling and school failure. This exploratory, descriptive and interpretative study, with a microetnographic touch, is based Bernstein's Sociolinguistic Codes Theory on to observe the tensions between national politics which opens the education to this immigrant population and the lack of preparation of teachers to face cultural changes in the mathematics classroom; revealing that one of the barriers that these students have is the establishment of an effective communication in the school environment.
\end{abstract}

Key words: Immigrant schooling, empowerment, school failure, school mathematics. 


\section{INTRODUCCIÓN Y ÁREA DE INVESTIGACIÓN}

Las matemáticas escolares son una de las áreas presentes en los currículos escolares de todos los países, muestra de ello son las pruebas estandarizadas nacionales e internacionales que miden este dominio del conocimiento; es en este contexto mundial donde se atribuye a las matemáticas la responsabilidad de desarrollar competencias que les permitan a los estudiantes pensar crítica y reflexivamente, tomar decisiones, entre otras, para su desenvolvimiento en el mundo actual (Valero, 2017).

En el contexto chileno nos encontramos con la prueba SIMCE (Sistema de Medición de la Calidad de la Educación) y la PSU (Prueba de Selección Universitaria), cuyos resultados ofrecen información respecto a las competencias que tienen los estudiantes, estableciendo el grado de alfabetización matemática y de otras áreas (Comité Técnico Asesor Consejo de Rectores de las Universidades Chilenas, 2010). A estas pruebas se les ha caracterizado -por el discurso popular- como promotoras del fracaso escolar (Dubet, 2019). Por otro lado, el entramado de la población en Chile se ha ido modificando, insertando una nueva variable, la 'inmigración'; esta puede mirarse como un elemento promotor del fracaso escolar debido a la descontextualización del currículo escolar entre países, o bien, la situación discriminatoria que sufren los estudiantes (Hernández, 2016). Enseguida se abordará de manera concisa el cambio que ha sufrido Chile, ya que hasta 1992 se había considerado un país migrante.

Entre 1992 y 2014, la cifra de migrantes residentes en Chile se cuadruplicó, caracterizada principalmente por mujeres de países como Perú, Argentina, Bolivia, Colombia y Ecuador; y se establecen, en su mayoría, en la Región Metropolitana y en las primeras tres regiones del país (Joiko y Vásquez, 2016). Además, las condiciones políticas y de pobreza que hoy viven algunos países de América, han impulsado la migración masiva de familias que ven a Chile como una oportunidad de equidad. De acuerdo con cifras oficiales, en el periodo 2013-2018, la población extranjera en Chile aumentó en más de un 31\% (Ministerio de Desarrollo Social, 2018), la mayoría provenientes de países de América Latina y el Caribe. Esta población contempla niños, niñas, jóvenes y personas adultas que continúan sus estudios en Chile; concentrándose en Tarapacá, Antofagasta, Arica y Parinacota, mientras que, en la Región de Los Lagos se registra una menor cantidad (Fernández, 2018).

En el 2012, el Gobierno de Chile se encontró con el desafío de poseer información precisa sobre la situación migratoria de los niños y niñas, y sus principales características, puesto que el desconocimiento de esto limitaba la información acerca de la inserción educacional, y como consecuencia, no se contaban con programas adecuados, ni con políticas y sistemas de seguimientos que protegieran los derechos de los estudiantes migrantes (Fernández, 2012).

Debido al incremento de la población extranjera, el Gobierno de Chile ha tenido que realizar un conjunto de mecanismos que permitan garantizar el ejercicio de aquellos derechos que el país ha consagrado para todos y todas, principalmente el de la Educación. Por esto, el Departamento de Extranjería y Migración del Ministerio del Interior y el Ministerio de Educación de Chile (MINEDUC), promueven la incorporación de los niños, niñas y jóvenes migrantes al sistema educativo, entregando facilidades para poseer una matrícula provisoria o definitiva en un establecimiento reconocido por el Estado; esto solo se aplicaba a los estudiantes y no modificaba la condición migratoria de su grupo familiar (Ministerio del Interior, 2003). Esta acción que apoyaba al estudiante extranjero permitió a 
las escuelas avanzar hacia la inclusión y lograr que niños y niñas accedieran al aprendizaje en un marco de respeto a la diversidad que representan (Fernández, 2018).

En general, los estudios sobre migración y educación en Chile expresan la relación entre escuela y familia, centrándose en las estrategias implementadas por las escuelas para promover una mejor relación entre ambas partes (e.g. Barrios-Valenzuela y Palou-Julian, 2014; Marín, 2015); además, se realizan diversas actividades extracurriculares por parte de las escuelas para incentivar la diversidad cultural que existe en los nuevos contextos escolares. Sin embargo, a pesar de la buena voluntad por parte del Estado por beneficiar a los escolares inmigrantes de su derecho humano para seguir estudiando, hay una serie de investigaciones que presentan la necesidad de generar un currículo intercultural, las cuales han tomado como principales sujetos de análisis al estudiante, el currículo y la familia (e.g. Aranda, 2011; Hernández, 2016; Pavez, 2013).

En el sentido anterior, trabajos como el desarrollado por Sanhueza, Paukner, San Martín y Friz (2012), sitúan la competencia de comunicación intercultural como una competencia que requiere ser desarrollada por el currículo escolar, ya que permite apoyar el proceso de inclusión, al dotar a los estudiantes de conocimientos, habilidades y actitudes que les permite apreciar la estética de una cultura, conectarse emocionalmente y desarrollarla. Esto no significa relacionarse con cada valor o práctica de una cultura, pero sí sentir un respeto saludable y una aceptación de su lógica interna. También consideran que,

debe proponer situaciones de aprendizaje que permitan a los estudiantes: (a) disfrutar de la interacción con personas de distinta cultura, $(b)$ desarrollar expectativas de poder y establecer buenas relaciones con personas de distinta cultura e (c) intercambiar experiencias, significados y cosmovisiones distintas (p. 36).

Respecto a los profesores, estos autores mencionan que, es necesario la incorporación de módulos o contenidos en las asignaturas del currículo sobre la comunicación intercultural desde una perspectiva interdisciplinar, diseñando instrumentos y programas específicos para diagnosticar y desarrollar la sensibilidad intercultural, entre otros. Por ejemplo, Sanhueza, Cardona y Friz (2012), realizaron un estudio sobre este tema a estudiantes de primaria y secundaria en Alicante, España. Los resultados evidencian que estos han tenido experiencia con compañeros de otra cultura, por lo que existe una gran aceptación y poseen una buena sensibilidad intercultural. Específicamente, las mujeres presentan una respuesta emocional positiva mayor que los varones hacia la relación intercultural, destacando actitudes de interés, respeto y compromiso con sus compañeros de clase culturalmente diferentes. Uno de los resultados obtenidos más significativos es que la sensibilidad cultural es mayor entre más pequeños, la que se va perdiendo con la edad, lo que se puede deber al cambio de prioridades y proyecto de vida que se genera con la edad, por ejemplo, la búsqueda de su identidad personal y social frente a sus pares. En esta etapa se produce un replanteamiento y una reestructuración de los valores, por lo que la influencia de los profesores es muy relevante.

En un trabajo similar al anterior, pero ahora apoyado en los profesores de Granada, Pomés y Sanhueza (2013), se explora la actitud de los profesores hacia la inclusión educativa, posicionando la premisa que, para que exista una transformación educativa en relación con la implementación de un sistema abierto a la diversidad, es necesario tener una especial atención al profesor, ya que éste es un agente clave y relevante para el proceso, al 
ser una barrera o un agente facilitador de las prácticas inclusivas. Existen diversos factores que influyen en las actitudes de los profesores hacia la inclusión educativa, relacionados con diversos aspectos:

a. La experiencia docente en el proceso de enseñanza-aprendizaje.

b. Las características de los estudiantes.

c. El tiempo del que se dispone para implementar acciones educativas se constituirá en otro factor relevante que impactaría la actitud del profesorado.

d. Los recursos con que se cuenta para facilitar el progreso del estudiante también podrían facilitar o limitar las posibilidades u oportunidades de generar espacios educativos más inclusivos.

e. Para que un profesor pueda tener una disposición frente a la atención a la diversidad y sea positiva, es necesario que pueda contar con la posibilidad de hacer adaptaciones curriculares, aplicar estrategias diversificadas de enseñanza y aprendizaje, entre otros.

Por último, los investigadores resaltan que una actitud positiva de los profesores apoya al proceso de inclusión, ofreciendo mejores oportunidades de desarrollo y participación de los estudiantes, al hacerse cargo de cada uno de sus miembros, innovando en sus políticas, cultura y práctica al interior de la comunidad para sostener una nueva manera de enfrentar el proceso educativo con mayor calidad.

A su vez, Infante (2010) señala los desafíos de la formación docente respecto a la inclusión educativa en los establecimientos educacionales; actualmente encontramos grupos heterogéneos marcados por diferencias en raza, género, lenguaje, estructura familiar, entre otros marcadores de identidades. Esta nueva configuración de las instituciones conlleva una diversidad en la que todos participan.

Este cambio ha implicado la transformación de los organismos gubernamentales haciendo énfasis en promover la inclusión en las escuelas. Todos estos factores plantean un desafío en la formación de los docentes para las instituciones de educación superior. Se necesita un profesional que pueda liderar las acciones educativas en relación con la diversidad e inclusión, que analice críticamente los propios sistemas de inclusión/exclusión y logre relacionarlos con la condición socioeconómica, formas de aprendizaje, nacionalidad, etnia, género, entre otros; que puedan repercutir en acciones de discriminación y opresión social, considerando aportes desde otras disciplinas y no sólo de la educación especial.

Esta investigación surge por el interés de determinar la mirada del profesor desde un sujeto de análisis diferente, situado en un área de conflicto, matemáticas, en donde ya existe un fracaso escolar, independiente de si hay o no población inmigrante. Por esto, nuestra área de interés se sitúa en el profesor de matemáticas que atiende en su aula a estudiantes extranjeros; así, desde el ámbito de la educación surge la pregunta fundamental del estudio: ¿cuáles son las necesidades y desafíos que percibe un profesor de matemáticas cuando atiende estudiantes extranjeros y busca evitar el fracaso escolar? Todo esto bajo el discurso del profesor, el que se explora mediante una entrevista semi-estructurada y un análisis microetnográfico; planificando los siguientes objetivos específicos de investigación:

a. conocer las áreas que el profesor prioriza en su discurso y los elementos que la componen, 
b. describir el tipo de necesidades que el profesor identifica en sus áreas prioritarias a partir de su experiencia, y

c. proponer líneas futuras de investigación en función a las áreas que el profesor de matemáticas prioriza a partir de su experiencia.

Como nos interesa analizar el discurso pedagógico del profesor de matemáticas sobre la población inmigrante presente en el aula, recurrimos al trabajo de Basil Bernstein, puesto que su teoría nos orienta con la premisa de que dicho discurso se apropia de otros discursos recontextualizados (Bernstein, 1982); situando este trabajo entre un análisis crítico de la realidad social externa y la representación interna en el aula.

\section{MARCO TEÓRICO}

La Teoría de los Códigos Sociolingüísticos de Basil Bernstein (Teoría del Déficit o Teoría Bernsteiniana de los Códigos) tuvo su motivación en el estudio de situaciones de deserción, fracaso escolar, rendimiento escolar, diferencias de aprendizaje en las distintas clases sociales, desigualdad a la movilidad, entre otras. En algunas ocasiones esta teoría ha sido mal interpretada, sosteniendo premisas de que existe déficit lingüístico en los sujetos de clases menos favorecidas. No obstante, formula una especie de privación sociocultural de tales sujetos con consecuencias de relaciones de tensión en su espacio socioeducativo, y el hecho de que el éxito escolar requiera un código elaborado con respecto al código dominante de la escuela; es decir, el déficit en el contexto tensiona las relaciones de macro poder (Bernstein, 1982).

Para Bernstein (1982, 1990), el currículo escolar resulta de una selección cultural basada en la construcción de conocimientos y el desarrollo de habilidades, aptitudes y actitudes. Cada país decide el acervo cultural de sus ciudadanos bajo los criterios de prioridad, considerando que el currículo que se construye, o que los elementos culturales que se van a considerar, otorguen a la ciudadanía ventajas significativas para mejorar su calidad de vida o su condición socioeconómica. En particular, cuando indagamos en el currículo nacional chileno bajo la óptica de Bernstein, se observa que el MINEDUC de Chile reconoce este nexo entre cultura y currículo, y establece que éste ofrece una base cultural común para todo el país, asegurando que la totalidad de los alumnos participe de una experiencia educativa similar y reciba un bagaje cultural compartido, favoreciendo su cohesión e integración social (Bases Curriculares de $7^{\circ}$ Básico a $2^{\circ}$ Medio) (Ministerio de Educación, 2015).

La forma en que una sociedad selecciona, clasifica, distribuye, transmite y evalúa el conocimiento educativo que considera público, refleja tanto la distribución del poder como los principios del control social (Bernstein, 1973). Hasta aquí, lo que hemos descrito de la teoría de Basil Bernstein, se enfoca en mostrar sus potencialidades como marco teórico para entender la reflexión dialéctica entre los conceptos contenidos en una teoría (lenguaje interno) y los datos empíricos que se pretenden analizar.

En nuestro estudio consideramos a la condición de inmigrante como una clase social a la manera de Bernstein (1982), es decir, como un elemento de estratificación que lo separa en un estrato social particular, en este caso de los ciudadanos chilenos, en donde identificamos que se dará lugar a un déficit lingüístico a este grupo social (escolares inmigrantes), fruto 
de las variables sociales que pueden desencadenar el fracaso escolar. Es aquí donde esta teoría regula dos tipos de códigos, el código restringido y el elaborado; los que guardan cierta similitud con el lenguaje público y el lenguaje privado. Nosotros proponemos que el profesor de matemáticas chileno, por su cercanía al caso de estudiantes inmigrantes, se mantendrá en un punto de equilibrio entre estos dos tipos de códigos sociolingüísticos. Un código elaborado proviene de su conocimiento sobre la política educativa y el currículo, pero que, a su vez, por su relación social con el fenómeno de inmigración, dará voz al código restringido que emerge de la cultura y subcultura que vive en la sala de clases, donde este código restringido es un ejemplo de su solidaridad apoyada en su experiencia individual de vida (Bernstein, 1989).

Por esto, para la operatividad del marco nos apoyaremos en tres conceptos claves como sugieren las investigaciones en educación, educación matemática y las bases curriculares (Ernest, 2002; Bases Curriculares de $7^{\circ}$ Básico a $2^{\circ}$ Medio, 2015; Torres, 2009; Salas, Castillo, San Martín, Kong, Thayer y Huepe, 2017; Terigi, 2009): el empoderamiento, la escolaridad inmigrante y el fracaso escolar. En seguida daremos voz a estos conceptos.

El empoderamiento se vincula a la institución humana con la capacidad de lograr sus objetivos. Es decir, es la toma de conciencia del estudiante sobre sus capacidades, desde la que puede potenciar su acción para transformar el mundo o transformarse (Torres, 2009). De acuerdo con Paul Ernest (2002) el empoderamiento al interior de la educación matemática se divide en tres dominios: el matemático, el social y el epistemológico.

a. El empoderamiento matemático consiste en ganar poder sobre el lenguaje, sobre los símbolos, las estrategias y las prácticas para utilizar un conocimiento específico de esta disciplina en la actividad de hacer matemáticas.

b. El empoderamiento social implicaría la toma de conciencia sobre la naturaleza de las matemáticas, es decir, entenderlas como un vehículo que permite reflexionar sobre el mundo.

c. El empoderamiento epistemológico hace referencia al crecimiento de la confianza en el uso de las matemáticas, en la creación y validación de este.

El término de escolaridad inmigrante (Salas et al., 2017) se usa para referirse a los escolares migrantes que, si bien tienen una situación legal o administrativa sobre su estatus socio territorial, comprende además su inserción a un contexto cultural diferente a aquel que usualmente compartía con su colectivo. Por esto, las propuestas de organismos como la UNESCO (2005), sugieren políticas para atender el desafío de la diversidad que están situadas en la atención a la diversidad, la educación intercultural, la vulnerabilidad de esta población. Pues las razones de exclusión que manifiestan los niños dentro de la escolaridad se deben a las tensiones existentes en los grupos respecto a sexo, raza, etnia y cultura; esto debido a que hay una gran valorización de origen en contraposición de los grupos culturalmente externos (Salas et al., 2017).

Por su parte, el fracaso escolar lo entenderemos como "la serie de dificultades que poco a poco van alejando al estudiante del ámbito escolar, impidiéndole terminar su formación" (Herrera, 1999, p. 4). Para la completa comprensión de este fenómeno es necesario mirar el fracaso escolar en términos psicoeducativos (Terigi, 2009), al identificarlo como un riesgo educativo que se establece entre los sujetos de un grupo sometidos a interacciones situacionales entre las prácticas escolares con las prácticas culturales específicas. 
En otras palabras, el fracaso escolar puede ser mirado como un riesgo educativo que pone de manifiesto la complejidad del tejido social que reproducen las interacciones sociales, situándolas en el sistema escolar, donde la actividad escolar reproduce conocimientos, habilidades y actitudes que se definen en un currículum; pero este último está lejano a orientar sobre prácticas culturales específicas que interaccionen de forma positiva o negativa durante la actividad escolar, acercando o alejando a los sujetos del conocimiento.

Para los fines de este trabajo, nos interesa observar la emergencia de categorías de análisis del código restringido versus el código elaborado del profesor de matemáticas; y a futuro, desarrollar una caracterización más fina de los elementos propuestos (empoderamiento, escolaridad inmigrante y fracaso escolar) que podrían considerarse líneas de acción para evitar el fracaso escolar en matemáticas de la población migrante en Chile.

\section{METODOLOGÍA}

\subsection{DISEÑO}

Esta investigación, de carácter exploratorio, descriptivo e interpretativo, se apoya en un estudio microetnográfico (Jiménez, Fardella y Muñoz, 2017), el que centra su análisis en patrones lingüísticos con los que interactúan los miembros de una comunidad educativa, hace énfasis en cuestiones sociolingüísticas y asume que la comunicación verbal y no verbal son culturalmente modeladas, aun cuando las personas en interacción no tengan conciencia de esto. Su propósito es el estudio de patrones en esta interacción; para el caso de esta investigación, se apoyó en una observación participante donde se grabó el audio de una entrevista semi-estructurada y se seleccionaron segmentos para el análisis detallado, que fueron codificados y analizados cualitativamente mediante en el programa Atlas ti.

\subsection{SUJETOS}

Los sujetos de este estudio son dos profesores de matemáticas de escuelas públicas, con población vulnerable, de la comuna de Osorno de la Región de Los Lagos, Chile, que atienden a estudiantes inmigrantes desde hace más de 5 años. El Profesor A, de 29 años e inserto en Educación Media, atiende estudiantes con edades entre 12 a 19 años; mientras que el Profesor B, de 32 años e inserto en Educación para Adultos, trabaja con estudiantes con edades entre 17 y 49 años. Estos profesores tienen jornada completa dentro de sus instituciones y generalmente dan servicio a grupos con más de 30 estudiantes, entre los que encuentran niños y jóvenes inmigrantes.

\subsection{INSTRUMENTO}

Se realizó una entrevista semi-estructurada a profundidad a lo largo de un par de sesiones, de 10 a 15 min., las que se llevaron a cabo en tiempos disponibles del profesor dentro de su institución de trabajo. La unidad de análisis se enfoca en identificar el significado privilegiado por el profesor de matemáticas, cuando se habla de inmigración en el salón de clase a partir de su vivencia. 
La entrevista semi-estructurada contenía en promedio 21 preguntas, divididas en 4 categorías, mismas que describiremos a continuación:

1. Nociones sobre el contexto. El primer bloque planteaba una serie de interrogantes acerca de las características contextuales de la escuela y su entorno; por ejemplo: ¿A qué tipo de sistema pertenece tu colegio? ¿Qué tipo de población recibe?

2. Conociendo la inmigración en tu colegio. En este bloque, las preguntas estaban dirigidas a conocer el manejo de las leyes migratorias por parte del profesor para la inserción de un estudiante extranjero en el sistema educativo e identificar el tipo de discurso sobre la política nacional posee; por mencionar: ¿Cuáles son los requisitos que necesita tener un niño extranjero para ingresar a su institución? ¿Cómo el estudiante extranjero revalida su nivel educativo?

3. Las estrategias pedagógicas implementadas. El tercer bloque de preguntas tenía como finalidad explorar las estrategias o herramientas didácticas que el profesor implantan para integrar al estudiante extranjero en la dinámica de la clase, así como las dificultades culturales o académicas que experimenta el profesor; para ejemplificar el tipo de interrogantes podemos mencionar: ¿Qué tipo de estrategias implementa para lograr la integración de los estudiantes extranjeros en el aula?

4. Reflexiones sobre la formación y práctica. Las preguntas del último bloque estaban dirigidas a indagar sobre la formación académica y la actualización que recibe el profesor para apoyar la integración de la población inmigrante en el aula; algunas interrogantes que se le hicieron al profesor fueron: ¿Considera usted que su formación académica le ha brindado herramientas que le permitan afrontar los desafíos que implica una enseñanza de la matemática para estudiantes extranjeros?

\subsection{ANÁLISIS DE DATOS}

El análisis de los datos recogidos de las entrevistas semi-estructuradas se dividió en dos fases. En la primera fase, se realizó una codificación ciega por expertos en Didáctica de las Matemáticas ajenos al estudio con el fin de evitar ambigüedades y subjetividad para identificar las unidades de análisis situadas en el código restringido y en el código elaborado; y en la segunda fase, mediante triangulación de expertos, se consensaron las unidades de análisis situadas en ambos tipos de códigos con el objetivo de extender las definiciones al hacer una caracterización más fina de estas.

\section{ANÁLISIS Y DISCUSIÓN}

Para el estudio sociolingüístico se recuperaron las unidades primarias de análisis presentes en el discurso del profesor (código elaborado), tales como el empoderamiento, la escolaridad inmigrante y el fracaso escolar; para cada una de estas unidades se identificaron -a lo largo de las grabaciones- palabras que bajo el discurso del profesor se asociaban a unidades secundarias o terciarias específicas (código restringido). A continuación, en la Figura 1, 2 y 3 se presentan las codificaciones ciegas hechas por los expertos (donde los investigadores no participaron). A partir de estas, se identificaron las unidades de análisis y se caracterizaron con mayor fineza por parte de los investigadores. 
Figura 1. Codificación del experto A

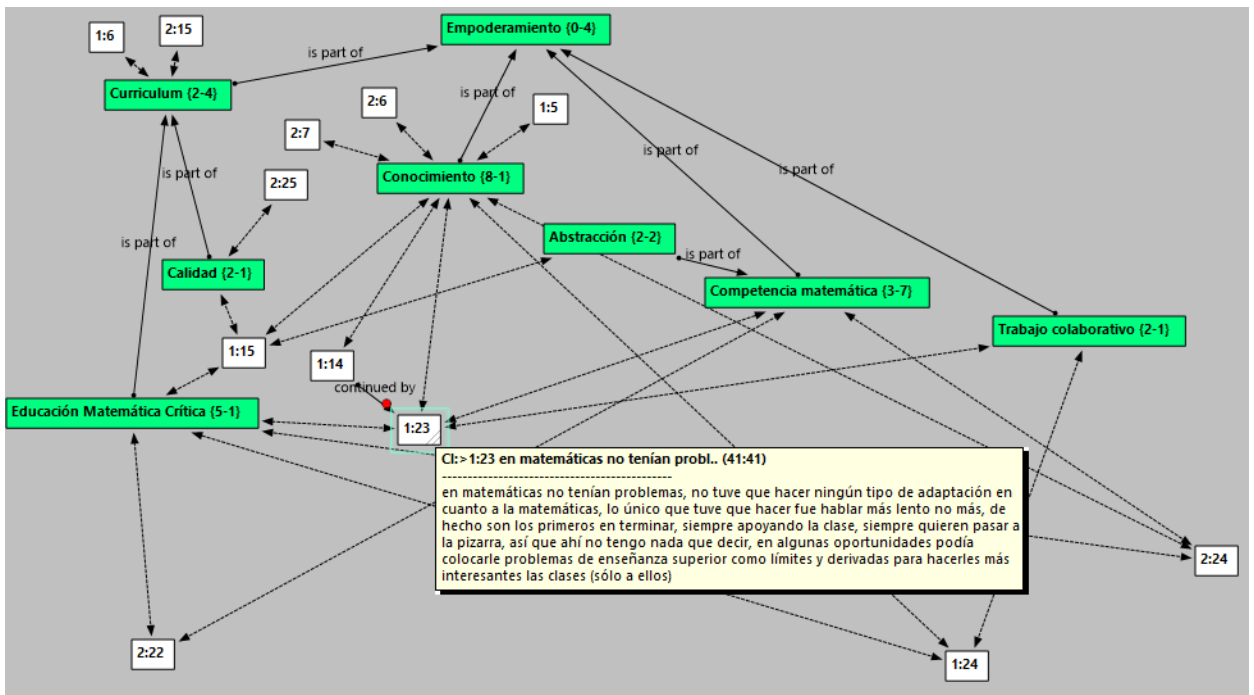

Figura 2. Codificación del experto B

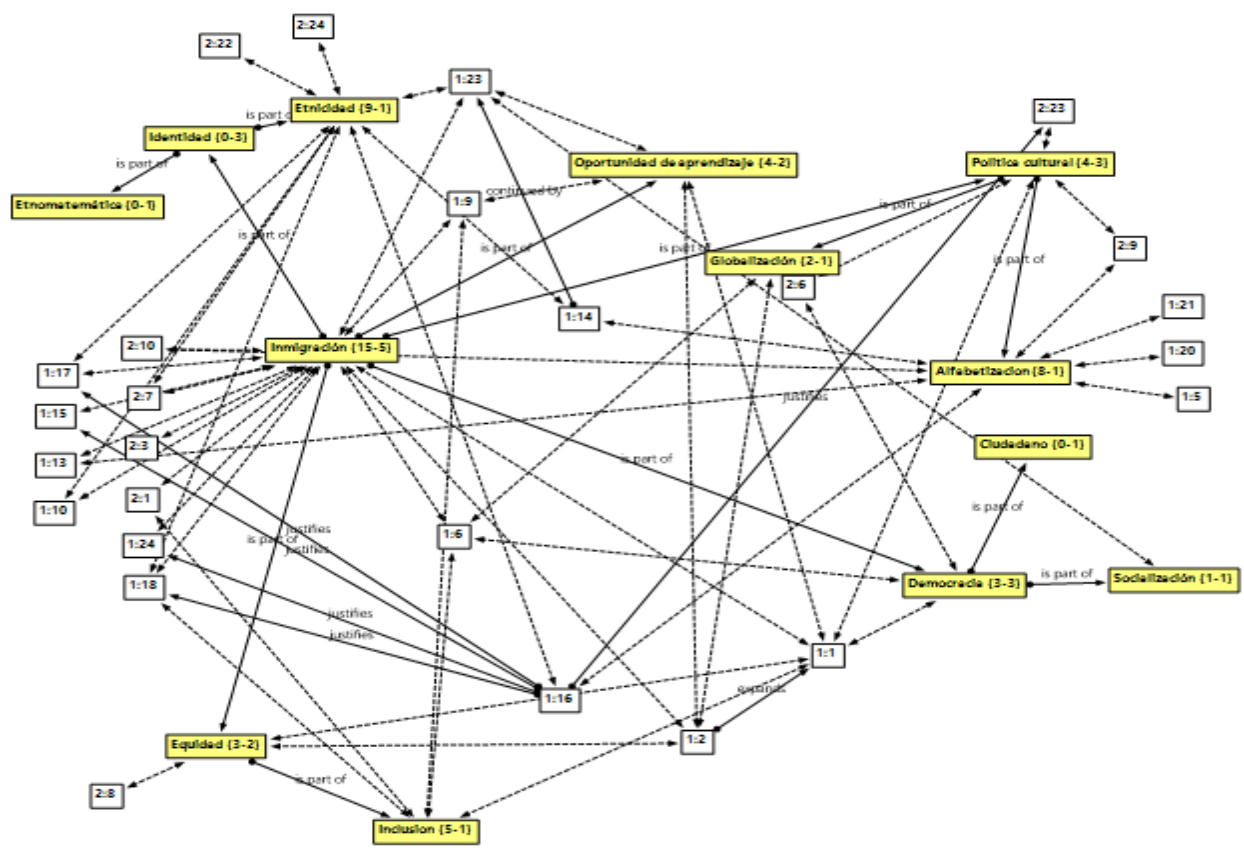


Figura 3. Codificación del experto C

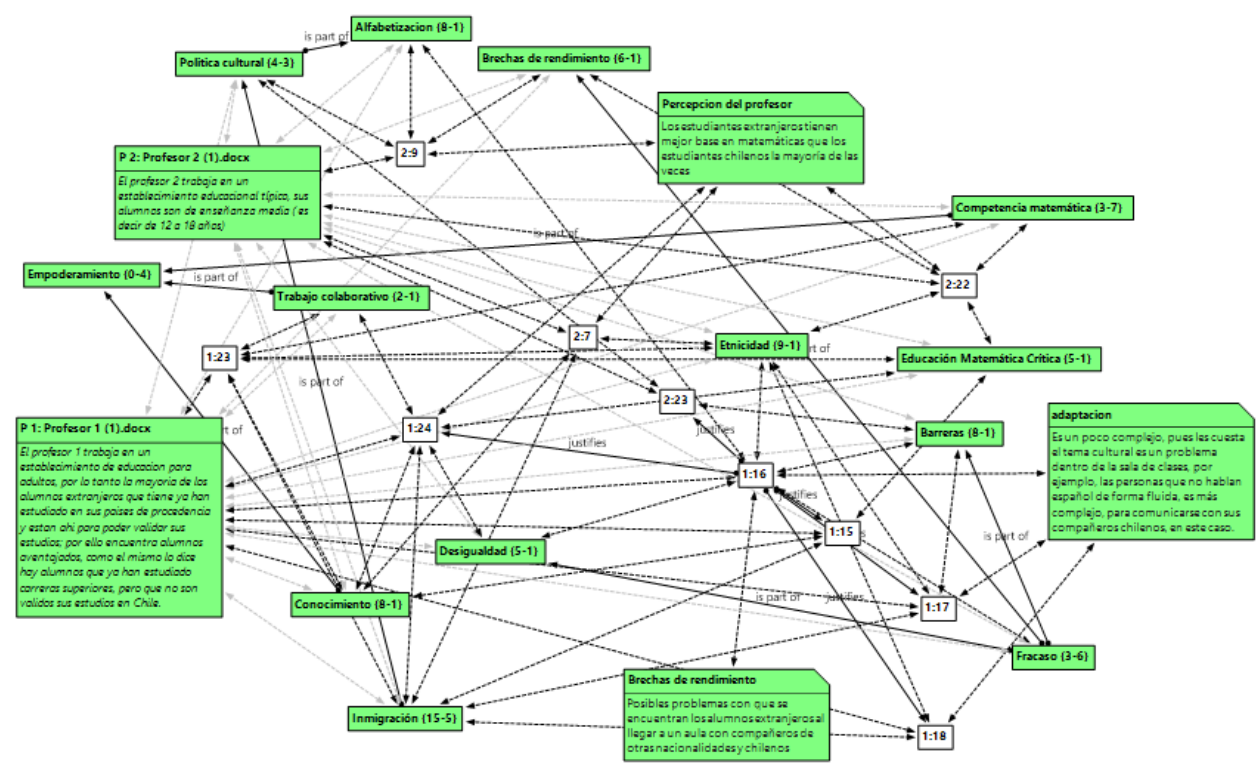

Una vez desarrollada la primera codificación ciega e identificación de las unidades de análisis, los investigadores llegaron a un consenso (triangulación de expertos) sobre las unidades sociolingüísticas, es decir, los códigos restringidos (unidad secundaria o terciaria) y los códigos elaborados (unidad primaria) en el discurso del profesor, los que guiaran la discusión y análisis de resultados (ver Figura 4).

Figura 4. Relaciones presentadas en el discurso del profesor

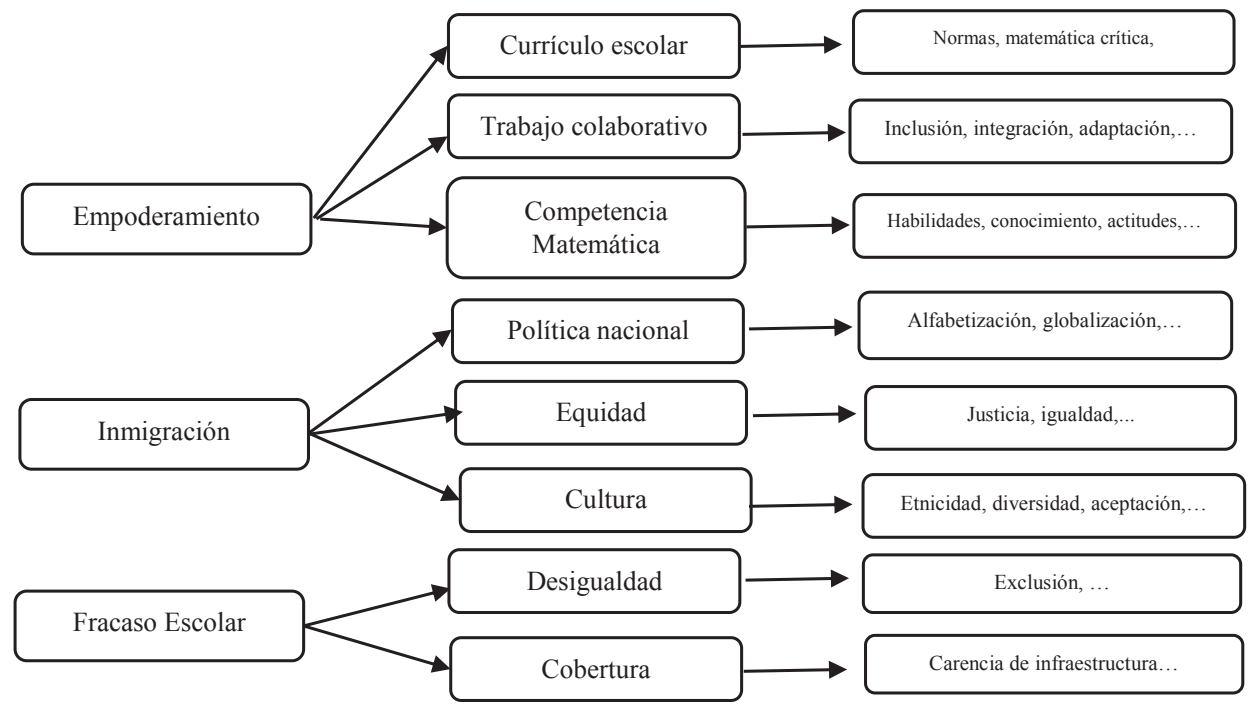


Enseguida, en la Figura 5 se presenta la red sociolingüística de los codificadores, desarrollada por consenso después de la triangulación de expertos, en donde se muestran los elementos desarrollados y presentados en la Figura 4.

Figura 5. Relaciones presentadas en el discurso del profesor

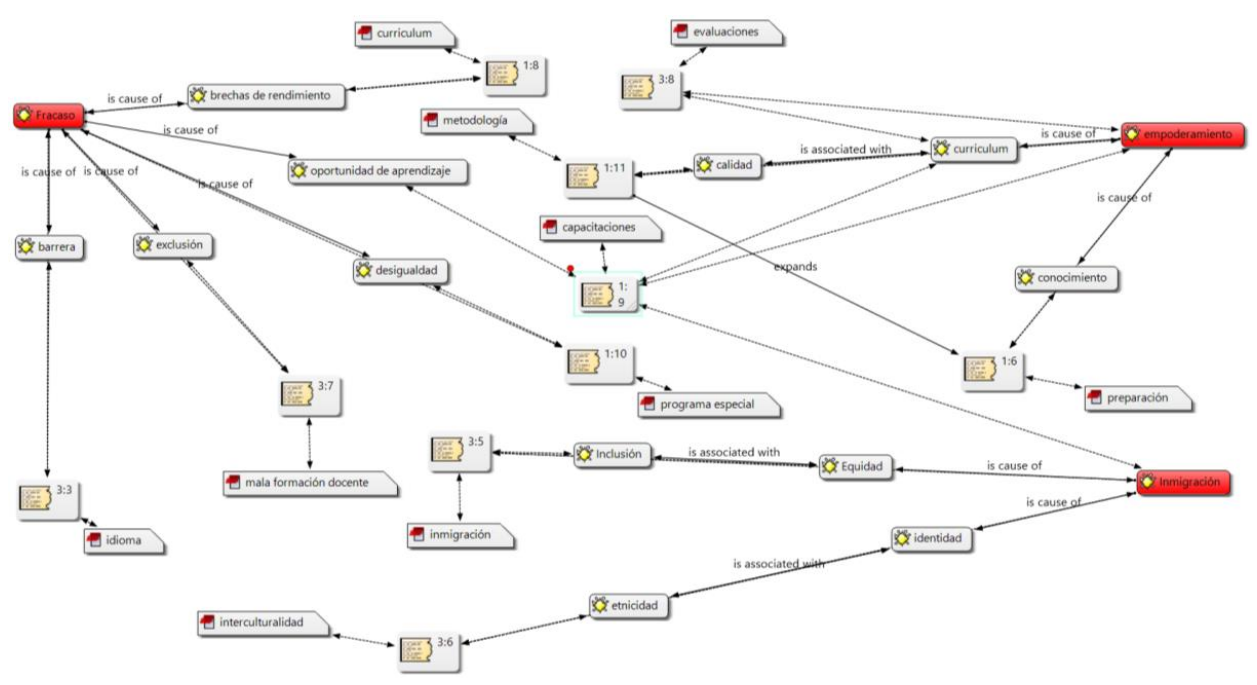

A continuación, se presenta el análisis global del discurso de los profesores dividido en tres etapas, determinadas por la reflexión de cada una de las unidades de análisis primarias (código elaborado) y la interrelación de estas, a partir del uso el software Atlas ti.

\subsection{ETAPA 1. EL EMPODERAMIENTO EN EL SALÓN DE MATEMÁTICAS}

El empoderamiento matemático que tiene el estudiante extranjero en las aulas chilenas se debe a una serie de prácticas sociales, donde pone a disposición del colectivo sus conocimientos, habilidades y actitudes matemáticas para interactuar con sus compañeros, y lograr un lugar que de otra forma le sería difícil obtener en el aula de matemáticas; ejemplo de esto, lo tenemos en los siguientes fragmentos:

En caso de matemáticas su adaptación es fácil, pues es operatoria básica, la cual manejan muy bien y rápido, además a sus compañeros les llama la atención que usan otras técnicas para multiplicar o dividir y muchos se acercan a querer a prender como lo hacen ellos, así hacen amigos (empoderamiento en lo social, profesor A).

Los estudiantes extranjeros tienen mejor preparación, se nota que hay un nivel mucho más alto en varios aspectos, ya sea culturales o cognitivos, se nota bastante, así que sus compañeros los buscan para que ellos los apoyen a resolver problemas, eso les permite socializar... el tema de la idiosincrasia, el chileno tiende a ser a veces muy 
discriminador y aísla en algunos casos, no siempre, pero aísla al estudiante extranjero (empoderamiento en lo social, profesor B).

Como puede notarse, el empoderamiento matemático que recibe el estudiante extranjero proviene de sus competencias matemáticas, de la interacción social a partir del trabajo colaborativo. Pero el currículo escolar también es benefactor en este proceso, no solo por el discurso político, sino por el tipo de contenidos que provee, como reflexionan enseguida los profesores:

me da la impresión de que ellos vienen con una muy buena base de los cursos más pequeños; lo que tiene que ver con, más que con conocimiento, con estructura, con hábitos de estudio, ... entonces, cuando les cuesta algo, ellos son como súper metódicos. En el caso de las niñas, estoy pensando en el caso de las niñas en específico, que yo he tenido; muy metódicas, muy muy metódicas. Y eso hace que pregunten, pregunten, pregunten; y van aprendiendo rápidamente, y esto las nivela, pero también les permite integrarse con sus compañeras, y que sus compañeras quieran trabajar con ellas (empoderamiento en lo curricular, profesor A).

Ellos en matemáticas no tienen problemas, como estas son más universales, incluso algunos de ellos están por encima de la media (empoderamiento en lo curricular, profesor B).

Como puede notarse en la entrevista de los profesores, ambos establecen que el empoderamiento matemático del estudiante extranjero es un eje articulador en la interacción social; el que es un punto para ser analizado en investigaciones futuras: ¿El empoderamiento matemático del estudiante extranjero puede apoyar a su integración sociocultural o requiere de más elementos? ¿El empoderamiento matemático del estudiante extranjero trasciende las paredes del aula o muere una vez terminada la actividad matemática?

\subsection{ETAPA 2: LA ESCOLARIDAD INMIGRANTEY SU PRESENCIA EN LA EDUCACIÓN MATEMÁTICA}

El proceso de inmigración que en los últimos años ha sufrido Chile, es un fenómeno al que la población no estaba acostumbrada; la mirada de los profesores sobre este fenómeno es observada desde tres frentes: la política, la equidad y la cultura.

Ambos profesores coinciden sobre tener conocimiento de los procesos migratorios que sufre su país; sin embargo, dicho proceso originalmente se centraba en la capital. Identifican que, desde el 2007, el flujo migratorio ha iniciado a modificar sus rutas y se intensifico en el 2014 hacia las regiones del sur, pues la población inmigrante observa en estas un espacio de crecimiento.

Dentro de los aspectos políticos y de equidad, los profesores coinciden en la existencia de una serie de normativas que permiten la incorporación de la población inmigrante en Chile; pero bajo su mirada, esta incorporación no es del todo clara y en algunos casos injusta, como se evidencia en el siguiente fragmento:

Los extranjeros que llegan generalmente hacen la nivelación en primero y segundo medio, después terminan obviamente tercero y cuarto. Por lo general están 2 años... 
Nosotros podemos hacer la prueba de nivelación de estudios, de hecho, el año pasado, a los estudiantes haitianos le hicimos nivelación de estudios de séptimo y octavo básico y de primero y segundo medio, para que ellos pudieran estar en tercero y cuarto medio. ¿Por qué hicimos eso? Porque la mayoría de los chicos que venían, venían con enseñanza secundaria aprobada completa, y la gran mayoría, como el 70\% había estado en instituciones de educación superior, entonces como en Chile no se le validaba ninguno de esos estudios, sino que los dejaban en séptimo básico o sexto básico, nosotros decidimos no hacer eso, y se pidió autorización al Ministerio de Educación, y el Ministerio de Educación accedió a que nosotros pudiéramos hacer ese tipo de sistema de entrada, que es único en Chile, porque no hay otra institución que lo haga (revalidación e injusticia, profesor B).

El fragmento anterior da cuenta de lo que en política educativa debe avanzar el Estado chileno; es difícil entender por qué los extranjeros de cierta nacionalidad, con todas las competencias mínimas necesarias para estar en igualdad de oportunidades, tienen dificultades para legalizar sus documentos académicos en correspondencia a otros extranjeros. Sin embargo, creemos que este es un proceso en el que se ha ido avanzando y donde hay aún mucho trabajo por hacer, porque si bien hay extranjeros a los que les es más fácil revalidar sus estudios, este proceso está regulado por el Ministerio de Educación, como se puede leer enseguida:

Ellos se reciben y después en el proceso, el Ministerio pide una validación de grado, que es una prueba que envía el mismo Ministerio (revalidación, profesor A).

Tienen que ir a la Provincial de Educación para ver el tema de convalidación de estudios y si su país tiene convenios educacionales con Chile, generalmente les convalidan la enseñanza básica o media. Si no los tienen, le hacen una ponderación de los años que han cursado en su país, y de acuerdo a eso, el Estado en realidad lo que hace es decirles: bueno el Estado le va a convalidar hasta tal año y usted debe hacer en Chile, no sé, tercero y cuarto medio, por ejemplo, en el caso de los colombianos y de los argentinos, generalmente se les convalida todos los años que traen de estudios, y por lo general, vienen a terminar tercero y cuarto medio la mayoría, pero en el caso de los haitianos, aunque ellos hayan tenido estudios superiores, acá en Chile, sólo le reconocían hasta 6to básico (revalidación, profesor B).

El proceso inmigratorio ha traído consigo cambios culturales, no solo en la interacción social, sino también en el aula de matemáticas, a los que el profesor debe estar atento y debe prepararse para atender, como resalta uno de nuestros entrevistados.

Hay cosas operatorias que los estudiantes extranjeros trabajan de forma diferente, por ejemplo, la forma de como acomodan la multiplicación, recuerdo haber visto la solución de un estudiante y no entenderla a simple vista, así que cuando el noto que lo observaba, solo sonreí, cuando tuve tiempo mire un video en YouTube y entendí su algoritmo, y ahora me siento preparado para corregir, así que cuando veo algo a lo que no estoy acostumbrado, lo anoto y lo reviso después para poder apoyar a mis estudiantes... (cambios culturales, profesor A). 
El fragmento anterior nos muestra la importancia de que existan ciclos de formación para los profesores, que les apoyen a sensibilizarse de los cambios culturales que sufre el salón al incorporar población inmigrante; porque si bien existe cursos en su formación continua sobre procesos de inclusión, estos no son específicos para dialogar sobre las dificultades que los profesores sufren al incorporar población migrante; sobre esta reflexión, se presenta el siguiente fragmento:

Sí, es necesario, tremendamente necesario. El tema es que se están dando muchas capacitaciones con respecto a la diversidad en general, atención a la diversidad en el aula, en toda la dichosa palabra. Pero yo pienso que es necesario tener capacitaciones, cursos, no sé, con respecto a específicamente al tema. No, por lo menos a mí no me ha tocado participar en alguna capacitación específica del tema. Como le digo, si he participado en cursos muy generales, muy amplios, diversidad en todos sus aspectos, pero específicamente en ese tipo de diversidad no sé, yo por lo menos no he participado y creo que es tremendamente necesario (capacitación, profesor A).

Esta necesidad de cursos debe plantear retos importantes en el trabajo dentro del área de las matemáticas desde diferentes aristas, por ejemplo, ¿cómo puede apoyar el profesor para que se genere la inclusión? ¿cuáles son los algoritmos, lenguaje o contenidos matemáticos que deben conocer los profesores de matemáticas para apoyar población migrante?, ¿qué nuevos desafíos plantean la formación inicial y continua de los profesores de matemáticas para apoyar a la población migrante?

Para los anteriores cuestionamientos, mostramos las reflexiones hechas por los entrevistados sobre su formación inicial y las estrategias que han usado para apoyar la integración de la población migrante.

La universidad no brinda herramientas para el trabajo de inclusión con extranjeros, se asume que uno va a trabajar con estudiantes 'normales' y el tema con estudiantes extranjeros o con estudiantes adultos, la universidad no lo mira, lo deja de lado o en realidad no se dan cuenta que existen (herramientas, profesor B).

Los chilenos hablamos muy rápido, ese es un problema, entonces les cuesta entender un poco a los extranjeros, lo que uno dice ya que tendemos a hablar demasiado rápido para ellos y tenemos demasiado modismo, entonces eso también les afecta, porque no utilizamos un vocabulario no tan formal, es un poco más coloquial, entonces colocamos un ritmo en la clase, al principio, después se adaptan y copian todos los modismos. Es decir, mi estrategia se fundamenta en hablar más despacio y fuerte (estrategias, profesor A).

Contextualizo los problemas en función a situaciones culturales cercanas a los estudiantes, entonces todos aprendemos, creo que hablar de historia y su cultura apoya a sensibilizar a todos, así que esa es mi estrategia al abrir la clase parte con preguntas cercanas a los estudiantes, para ello antes les pregunto de dónde vienen y que hacían y trato de adaptar los problemas (estrategias, profesor B).

Como se puede notar en las reflexiones de los profesores A y B, muchas de sus estrategias surgen de su experiencia personal y de sus creencias, pero no están fundamentadas en 
posturas teóricas existentes, lo que hace plantearnos interrogantes como: ¿qué proporción de las estrategias usadas por los profesores de matemáticas se apoyan en marcos teóricos?, ¿qué características tienen las estrategias que usa el profesor de matemáticas para la atención inmigrante? ¿cómo puede potencializarse el uso de los aspectos históricos de las poblaciones inmigrantes?

\subsection{ETAPA 3: EL FRACASO ESCOLAR EN LA POBLACIÓN INMIGRANTE}

Con relación al fracaso escolar, los profesores lo ubican en dos terrenos: uno situado en la cobertura y otro en la desigualdad; sobre esto, presentamos dos fragmentos de las entrevistas de los profesores:

Es complicado para los que viven en las afueras desplazarse a escuelas y más si están trabajando sus padres o ellos deben trabajar, la ventaja que tenemos es ofrecer desayunos (fracaso, profesor A).

Es complejo dentro de la sala de clases, por ejemplo, a las personas les cuesta trabajo comunicarse con sus compañeros chilenos, interactuar, en especial a los haitianos, es muy difícil su idioma el creole, aun cuando son muy sonrientes y participativos. Por ejemplo, con los colombianos, ellos son cerrados y les cuenta trabajo tratar con los modismos a veces se ofenden y ellos son los que más renuncian a terminar... yo me he dado cuenta de que es un problema, el tema del idioma, esa es como la barrera (fracaso, profesor B).

El fracaso escolar situado por los profesores representa un reto, el más fuerte es el idioma, pues en algunos casos (haitianos) es una limitante por no entenderlo, y en otros (colombianos, mexicanos, venezolanos) puede tener una connotación de tipo socio cultural al no comprender el significado de algunas palabras en el mismo idioma. Enseguida se dialogará sobre las conclusiones de este estudio exploratorio y las líneas de investigación futuras que se plantean como reto en la Educación Matemática.

\section{CONCLUSIONES Y LÍNEAS FUTURAS DE TRABAJO}

Después del análisis anterior, observamos que el profesor de matemáticas es un mediador entre el código elaborado proveniente de la política nacional y el currículo, y el código restringido supeditado en su vivencia con la población inmigrante. Para ello, este estudio identifica a partir del discurso de profesor tres áreas de interés en el discurso, a saber, el empoderamiento matemático, el fracaso escolar y la escolaridad inmigrante; y provee de algunos rasgos de estos códigos, fundamentados en los códigos restringidos.

También se identificó que bajo la mirada del profesor de matemáticas (Granada et al., 2013; Sanhueza et al., 2012) el éxito del estudiante extranjero en el sistema educativo chileno depende en gran medida de situaciones socioculturales de adaptación a la idiosincrasia chilena, en particular, identifican bajo su experiencia que un elemento que hace fracasar al estudiante extranjero depende del flujo de una comunicación efectiva entre el alumno extranjero y el docente o entre los alumnos, y no una inadecuada formación académica de los estudiantes extranjeros. 
Además, los profesores entrevistados coinciden que la formación del docente es débil en temas de inclusión (Sanhueza et al., 2012), en específico de estudiantes extranjeros. También, ambos concuerdan con la necesidad de revisar el currículo chileno de matemáticas, el que consideran muy amplio e imposibilita el buen manejo de algoritmos matemáticos por parte de estudiantes nacionales y extranjeros; y lo importante de generar un currículo intercultural para el profesor de matemáticas, de manera que pueda motivar emocionalmente a los estudiantes extranjeros y que apoye a tener un mayor número de representaciones matemáticas, en particular, logre conocer los diferentes tipos de algoritmos que se usan alrededor del mundo, con esto puede el estudiante tener una alfabetización matemática que le permita competir en el mundo globalizado.

Por otro lado, los profesores destacan que la inclusión de la cultura puede ser un motor en el salón de matemáticas (Infante, 2010); esto nos conlleva a establecer algunas preguntas que guíen futuras líneas de investigación, tales como: ¿Es necesaria una modificación curricular en Chile para que ésta contemple a la población extranjera y su interculturalidad? ¿La formación inicial y continua de docentes debe establecer temas de inclusión con extranjeros? ¿Cómo puede la historia y la cultura generar espacios de inclusión en la sala de matemáticas? ¿El empoderamiento del estudiante extranjero en el aula de matemáticas, como puede llevarse a fuera de ella para lograr su total adaptación al sistema educativo y cultural chileno?

Todo esto para el desarrollo de una caracterización de códigos restringidos y elaborados que manifieste un profesor de matemáticas, de forma que en el futuro esta información apoye para generar nuevas propuestas de equidad en la sala de matemáticas.

\section{REFERENCIAS BIBLIOGRÁFICAS}

Aranda, V. (2011). Reflexión y análisis de políticas y prácticas innovadoras a la luz de las representaciones sociales y de la necesidad de una educación intercultural en la formación inicial docente. Estudios Pedagógicos, 37(2), 301-314.

Barrios-Valenzuela, A. y Palou-Julian, B. (2014). Educación intercultural en Chile: la integración del alumnado extranjero en el sistema escolar. Educación y Educadores, 17(3), 405-426.

Bernstein, B. (1973). Applied Studies Towards a Sociology of Language. Londres, Inglaterra: Taylor $\&$ Francis.

. (1982). Pedagogía, control simbólico e identidad. Madrid, España: Ediciones Akal.

. (1989). Estudios teóricos para una sociología del lenguaje. Madrid, España: Ediciones Akal. . (1990). The structuring of pedagogic discourse. Londres, Inglaterra: Taylor \& Francis.

Comité Técnico Asesor Consejo de Rectores de las Universidades Chilenas (2010). Resultados de la vinculación Prueba SIMCE 2006 y PSU Admisión 2009. Santiago, Chile: Ministerio de Educación.

Dubet, F. (2019). Escuelas actuales generan injusticias. Seminario de la Facultad de Educación. Ponencia llevada a cabo en la Pontificia Universidad Católica de Chile, Santiago, Chile. Recuperado el 9 de marzo de 2019 de: http://educacion.uc.cl/listado-de-noticias/687-francoisdubet-escuelas-actuales-generan-injusticias.

Ernest, P. (2002). Empowerment in mathematics education. Philosophy of Mathematics Education Journal, 15(1), 1-16.

Fernández, M. (2018). Mapa del estudiantado extranjero en el sistema escolar chileno (2015-2017). Santiago, Chile: Ministerio de Educación.

Fernández, R. (2012). Una mirada a los derechos de los niños, niñas y adolescentes inmigrantes en 
Chile. Avances y desafíos pendientes. En A. Riva, D. Lawson, Y. Ocon, S. Lahoz, N. Espejo, A. Barlat (Eds.), Una mirada a los derechos de los niños, niñas y adolescentes inmigrantes en Chile. Avances y desafíos pendientes (pp. 243-273). Santiago, Chile: Fondo de las Naciones Unidas para la Infancia/UNICEF.

García, P., Hernández, Á., Gomáriz, Á. y Parra, J. (2016). Familia, inmigración y comunicación con el centro escolar: un estudio comparativo. Educación XXI, 19(2), 127-151.

Granada, M., Pomés, M. y Sanhueza, S. (2013). Actitud de los profesores hacia la inclusión educativa. Centro de Estudios Interdisciplinarios en Etnolingüística y Antropología Socio-Cultural, (25), 51-59.

Hernández, A. (2016). El currículo en contextos de estudiantes migrantes: Las complejidades del desarrollo curricular desde la perspectiva de los docentes de aula. Estudios pedagógicos, 42(2), 151-169.

Herrera, M. E. (1999). Fracaso escolar, códigos y disciplina: una aproximación etnográfica. Última década, 7(10), 126-141.

Infante, M. (2010). Desafíos a la formación docente: inclusión educativa. Estudios pedagógicos, 36(1), 287-297.

Jiménez, F.; Fardella, C. y Muñoz, C. (2017). Una aproximación microetnográfica de prácticas pedagógicas en escuelas multiculturales. Tensiones y desafíos en torno a la escolarización de inmigrantes y grupos minoritarios. Perfiles Educativos, 39(156), 72-82.

Joiko, S. y Vásquez, A. (2016). Acceso y elección escolar de familias migrantes en Chile: No tuve problemas porque la escuela es abierta, porque acepta muchas nacionalidades. Calidad en la educación, (45), 132-173.

Ministerio de Desarrollo Social (2018). Flujos de población en el territorio. Perfil y tipología de la migración inter-regional e inter-comunal. Informe $N^{\circ} 8$ Ministerio de desarrollo Social. Santiago, Chile: Ministerio de Desarrollo Social.

Ministerio de Educación (2015). Bases Curriculares $7^{\circ}$ básico a $2^{\circ}$ medio. Santiago, Chile: Ministerio de Educación.

Ministerio del Interior. (2003). Circular $N^{o} 1179$, de 28 de enero de 2003. Santiago, Chile: Ministerio del Interior.

Pavez, I. (2013). Los significados de ser niña y niño migrante: conceptualizaciones desde la infancia peruana en Chile. Revista Polis, 12(35), 1-19.

Salas, N.; Castillo, D.; San Martín, C.; Kong, F.; Thayer, L. y Huepe, D. (2017). Inmigración en la escuela: caracterización del prejuicio hacia escolares migrantes en Chile. Universitas Psychologica, 16(5), 1-15.

Sanhueza, S.; Cardona, C. y Friz, M. (2012). La sensibilidad intercultural en el alumnado de educación primaria y secundaria de la provincia de Alicante. Perfiles educativos, 34(136), 8-22.

Sanhueza, S., Paukner, F., San Martín, V. y Friz, M. (2012). Dimensiones de la competencia comunicativa intercultural (CCI) y sus implicaciones para la práctica educative. Folios, (36), 131-151.

Terigi, F. (2009). El fracaso escolar desde la perspectiva psicoeducativa: hacia una reconceptualización situacional. Revista iberoamericana de educación, 50, 23-39.

Torres, A. (2009). La educación para el empoderamiento y sus desafíos. Sapiens: Revista Universitaria de Investigación, 10(1), 89-108.

Valero, P. (2017). Mathematics for all, economic growth, and the making of the citizen-worker. En T. S. Popkewitz, J. Díaz y C. Kirchgasler (Eds.), A political sociology of educational knowledge: Studies of exclusions and difference (pp. 117-132). New York, USA: Routledge. 
